\title{
Non-operative arterial embolisation in primary liver tumours
}

\author{
P G WHEELER, W MELIA, P DUBBINS, B JONES, H NUNNERLEY, P JOHNSON, \\ ROGER WILLIAMS
}

British Medical fournal, 1979, 2, 242-244

\section{Summary and conclusions}

Three patients with primary hepatic tumours were treated by selective arterial embolisation with gelatinfoam fragments to induce necrosis. In the two with histologically proved hepatocellular carcinoma ultrasonography suggested that necrosis had been induced, as did the rapid initial falls in serum $\alpha$-fetoprotein concentration by 95 and $81 \%$ of the original values respectively. Treatment was continued with a course of adriamycin, and both patients remained well and symptom free at 10 and 12 months. In the third patient, who had an expanding and highly vascular benign hepatic adenoma associated with use of a contraceptive pill, embolisation obliterated the tumour mass.

Tumour embolisation should be regarded as only the first step in managing hepatocellular carcinoma and as a means of reducing appreciably the viable tumour mass before chemotherapy. It may be used as the primary and definitive treatment in patients with benign liver tumours.

\section{Introduction}

Primary and secondary hepatic neoplasms derive most of their blood supply from the hepatic artery, ${ }^{1}$ and devascularisation by surgical ligation of this vessel has been used to induce necrosis in such tumours. An appreciable palliative effect in both hepatocellular carcinoma and metastases from primary intestinal tumours has been reported. ${ }^{2}{ }^{3}$ To ensure complete devascularisation, ligation has to be carried out close to the liver hilum. This may be technically impossible when the liver is massively enlarged, and collaterals from most of the neighbouring arteries form rapidly, often by four to six weeks, presenting another problem, and the tumour masses may begin to increase in size again after only a few months. More distal occlusion, by injecting embolic material into the peripheral arteries at the time of arteriography, is currently being carried out for tumours in other organs, and we thought that this might be useful in cases of hepatic primary carcinoma. The chance of collateral formation is reduced, the technique repeatable, and selective catheterisation and devascularisation of individual tumours possible. We here describe the use of this method in three patients, two with a primary hepatocellular carcinoma and one with a highly vascular hepatic adenoma associated with oral contraceptive use.

Liver Unit, King's College Hospital and Medical School, London SE5 8RX

P G WHEELER, DM, MRCP, lecturer

W MELIA, MB, MRCP, research fellow

B JONES, MB, MRCP, registrar

P JOHNSON, MB, MRCP, research fellow

ROGER WILLIAMS, MD, FRCP, director and consultant physician

Department of Diagnostic Radiology, King's College Hospital

$P$ DUBBINS, MB, FRCR, senior registrar

H NUNNERLEY, MB, FRCR, consultant radiologist

\section{Patients and methods}

In cases 1 and 2 hepatocellular carcinoma was firmly diagnosed on direct needle biopsy of the tumour mass. Both patients had considerably raised serum $\alpha$-fetoprotein (AFP) concentrations. One patient (case 1) had noticed an enlarging lump in the epigastrium over the previous two months, and the other (case 2) presented with a three-month history of epigastric pain and weight loss. Both had clinical hepatomegaly, particularly of the left lobe, and in both isotope liver scanning and hepatic grey-scale ultrasonography showed a left lobe filling defect. Both patients had serum positive for hepatitis B surface
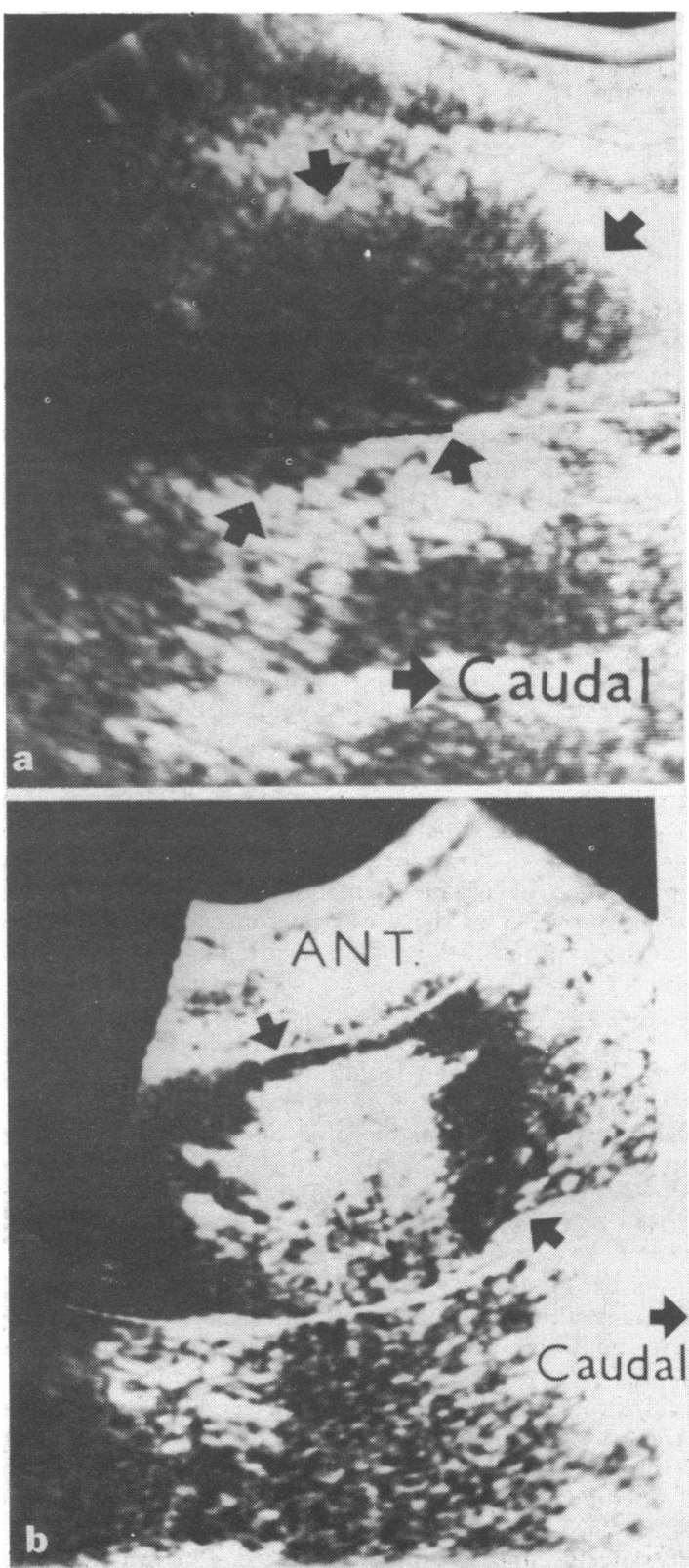

FIG 1-Case 1. Hepatic grey-scale ultrasound scan (longitudinal through left lobe) before $(a)$ and after $(b)$ therapeutic embolisation. Black arrows outline left lobe hepatoma. 
antigen, and in case 1 the non-tumorous part of the liver showed evidence of an underlying cirrhosis. The patient in case 3 , who had noticed a painless but enlarging epigastric mass for 18 months, had been continuously taking an oral contraceptive pill for seven years. Exploratory laparotomy at the referring hospital disclosed a large vascular tumour in the right lobe of the liver, which biopsy showed to be a benign adenoma. Isotope liver scanning using ${ }^{99} \mathrm{~m}$ technetiumlabelled sulphur colloid showed the unusual appearance of an area of greatly increased uptake in the right lobe, corresponding to the site of the tumour.

Arterial embolisation was carried out under general anaesthesia in cases 1 and 2 and local anaesthesia in case 3 immediately after diagnostic arteriography. Fragments of gelatin foam (Sterispon), cut into cubes about $2 \times 2 \times 4 \mathrm{~mm}$, were packed into $1 \mathrm{ml}$ tuberculin syringes in a suitable contrast medium such as Hypaque $45 \%$. After selective catheterisation of the supplying artery the contents of about eight of these syringes were injected down the catheter, each followed by a flushing injection of contrast medium or saline. The injection was continued until an immobile column of contrast-soaked gelatin foam was seen to fill this vessel and not to move distally between injections (see fig 3(b)).

The patients in cases 1 and 2 were covered against opportunistic anaerobic infection with benzylpenicillin and metronidazole, starting one hour before the procedure and continuing for 10 days.

\section{Results}

PATIENTS WITH HEPATOCELlULAR CARCINOMa (cases 1 and 2)

The free flush and coeliac-axis arteriograms in case 1 showed the left hepatic lobe tumour to be supplied by a separate branch of the coeliac axis. This artery was selectively catheterised and occluded by embolisation. In case 2 a single large vascular tumour was supplied by the left and middle hepatic arteries, which were separately catheterised and successfully occluded. The right lobe of the liver was supplied from the superior mesenteric artery. The portal vein was occluded in case 2 but patent in case 1 . In neither case was there arteriographic evidence of tumour elsewhere in the liver. Within 24 hours after the procedure both patients complained of abdominal pain, which required analgesia with pethidine for up to 10 days. Serum aspartate aminotransferase, alkaline phosphatase, and bilirubin

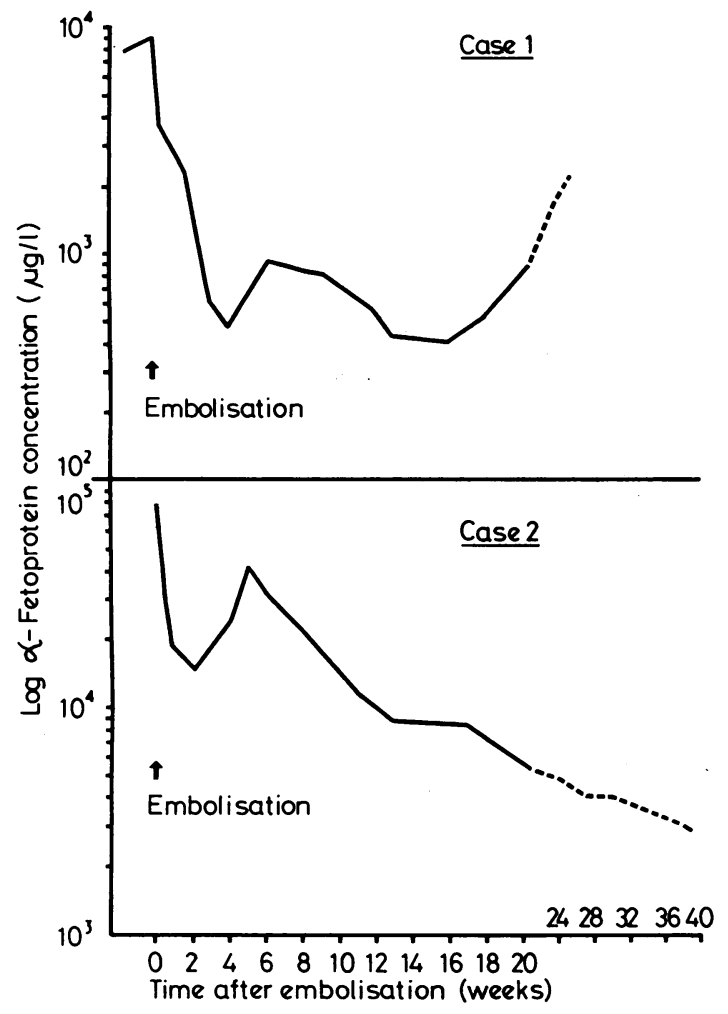

FIG 2 -Serial measurements of log serum $\alpha$-fetoprotein concentration in cases 1 and 2 after therapeutic embolisation.

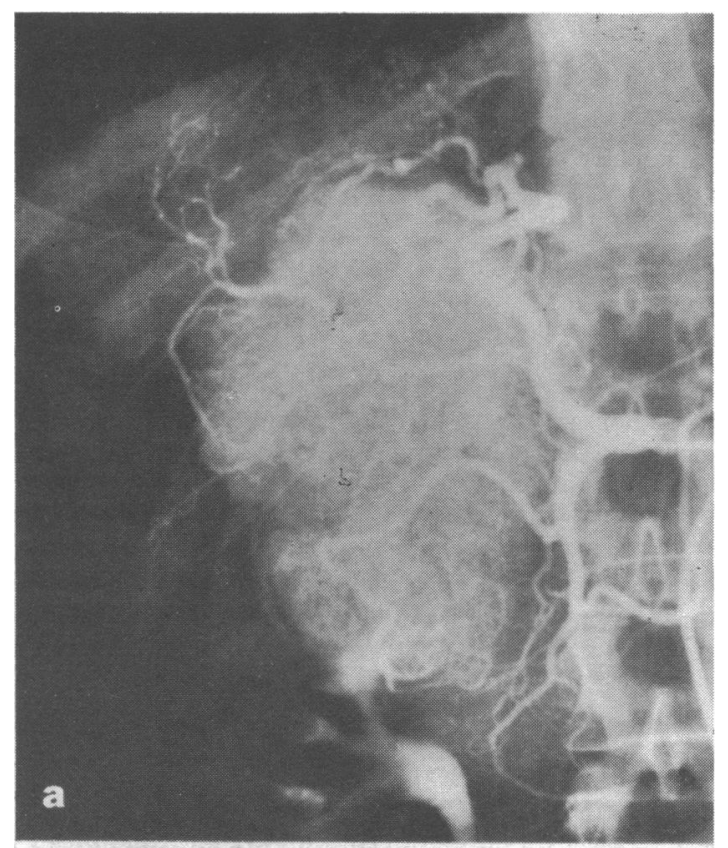

concentrations rose in both cases but had returned to pretreatment values within two weeks.

The size of the tumour appeared to increase slightly after the first week in both cases, as shown by serial grey-scale ultrasonography, possibly owing to oedema. In case 1 the tumour size subsequently decreased by $13 \%$ of the original diameter in two weeks, and pronounced central echogenicity became apparent, which would be compatible with areas of necrosis (fig 1 ). In case 2 the tumour diameter decreased less (an estimated $5 \%$ in one month). The serum AFP concentrations, however, fell appreciably: in case 1 by $95 \%$ of the original value in four weeks and in case 2 by $81 \%$ in two weeks (fig 2). The greatest falls were in the first week, being $71 \%$ and $76 \%$ respectively, and the first decrease occurred between one and two days after the procedure.

After four weeks in case 1 and two weeks in case 2 the serum AFP concentrations began to increase (fig 2). At this point treatment was started with intravenous adriamycin at a dose of $60 \mathrm{mg} / \mathrm{m}^{2}$ threeweekly, and the concentrations again decreased over the next eight weeks to values below those achieved by embolisation. In case 1 there was a further increase at four months, and treatment was changed to 
a new chemotherapeutic agent, VP 16/213 (etoposide). The patient remained clinically well 12 months after embolisation. In case 2 AFP concentrations continued to fall steadily over a total of 10 months to a value $3 \%$ of that at presentation, and the tumour diameter, as shown by ultrasonography, decreased by $31 \%$ overall. The epigastric mass became impalpable and the serum alkaline phosphatase concentration only slightly raised (115 IU/l as compared with $690 \mathrm{IU} / 1$ previously).

\section{PATIENT WITH BENIGN ADENOMA (case 3)}

Hepatic arteriography showed a well-circumscribed and highly vascular tumour in this patient. Complete occlusion of its arterial supply by embolisation was shown arteriographically (fig 3 ). Within 24 hours the patient developed nausea and vomiting and some upper abdominal pain lasting about a week. Subsequent recovery was delayed by the development of an abscess cavity, but after surgical drainage she improved rapidly. Repeat hepatic arteriography at four weeks confirmed that the distal portion of the main hepatic artery was stil occluded, and follow-up at 12 months, including isotope scanning, showed no evidence of any remaining adenoma.

\section{Discussion}

The changes in serum AFP concentration leave no doubt that the objective of reducing viable tumour size was achieved in the two cases of hepatocellular carcinoma. Changes in AFP concentration are proportional to changes in viable tumour cell mass. ${ }^{4}$ Although the shrinkage in overall tumour size was small, as estimated by ultrasonography, the central echogenicity that developed would also be consistent with the production of tumour necrosis.

Embolisation has obvious advantages for the patient, since surgery is avoided and greater devascularisation is almost certainly achieved than with surgical ligation. Moreover, selective embolisation of individual tumours is possible, as in our two patients with hepatoma, in neither of whom would hepatic artery ligation have been feasible because of cirrhosis in the first and a portal vein block in the second. The pain after the procedure may be quite severe, though may be controlled with pethidine, and may occur partly because the liver capsule is affected by more peripheral embolisation. The abscess in the patient in case 3, who underwent embolisation first, may have formed because prophylactic antibiotics were omitted; the eventual outcome was, nevertheless, highly satisfactory. Other reported complications, which did not occur in our patients, are inadvertent embolisation of normal structures and passage of emboli into the lungs through arteriovenous shunts. ${ }^{5}$

Tumour embolisation, as with surgical ligation, should be regarded as only the first step in managing hepatocellular carcinoma and as a means of reducing appreciably the viable tumour mass before chemotherapy. The effect of embolisation and the timing of chemotherapy may be well assessed by serial measurement of serum AFP concentrations. Although pain was not a prominent feature in our cases, embolisation may alleviate it considerably. ${ }^{6}{ }^{7}$ Embolisation has also been used for metastatic endocrine-hormone-secreting tumours in the liver, such as carcinoids and glucagonomas, ${ }^{8}$ reducing the circulating hormone concentrations considerably. In patients with benign liver tumours, as in case 3, embolisation may be used as the primary and definitive treatment. These tumours may regress after oral contraceptives have been withdrawn, but in patients in whom they do not or in whom complications such as intraperitoneal haemorrhage or pain occur, more active treatment measures become necessary. The alternative procedure to embolisationnamely, surgical resection-is by comparison a major undertaking.

We are indebted to the Cancer Research Campaign for generous support and to Linda Brookes for editorial help.

\section{References}

${ }^{1}$ Breedis, C, and Young, G, American Fournal of Pathology, 1954, 30, 969.

${ }^{2} \mathrm{Kim}, \mathrm{D} \mathrm{K}$, Kinne, D W, and Fortner, J G, Surgery, Gynecology and Obstetrics, 1973, 136, 966.

${ }^{3}$ Murray-Lyon, I M, Fournal of the Royal College of Surgeons of Edinburgh, $1972,17,156$.

4 Johnson, P J, et al, Lancet, 1978, 1, 1006.

5 Allison, D J, British Fournal of Hospital Medicine, 1978, 20, 707.

${ }^{6}$ Goldstein, H M, et al, Radiology, 1976, 120, 539.

${ }^{7}$ Dick, R, British fournal of Radiology, 1977, 50, 241

${ }^{8}$ Allison, D J, Modlin, I M, and Jenkins, W J, Lancet, 1977, 2, 1323.

(Accepted 15 fune 1979)

\section{SHORT REPORTS}

\section{Emboli from a prosthetic heart valve during postmenopausal oestrogen therapy}

While the possible thromboembolic complications of oestrogencontaining oral contraceptives have received wide publicity, ${ }^{1}$ the risks of similar complications during oestrogen therapy for control of menopausal symptoms may be overlooked. We report a case of multiple systemic emboli occurring during oestrogen therapy in a patient with a prosthetic mitral valve.

\section{Case report}

A 52-year-old postmenopausal woman with rheumatic heart disease had undergone mitral valve replacement with a Starr-Edwards prosthesis in 1971, after the development of severe mitral regurgitation through a previously inserted fascia lata valve. Recovery was uncomplicated. At regular follow-up visits over the next seven years she remained in atrial fibrillation with no evidence of dysfunction of the valve prosthesis or of cardiac failure. Her only symptom was mild exertional dyspnoea. She was maintained throughout on digoxin $0.25 \mathrm{mg}$ daily, frusemide $40 \mathrm{mg}$ daily with potassium supplements, and supervised anticoagulation with phenindione.
During 1978 she developed recurrent urinary tract infections and was referred to a urologist. As the urinary infections were attributed to postmenopausal atrophic changes she was given conjugated equine oestrogens (Premarin) $0.625 \mathrm{mg}$ twice daily. Several days later she experienced a sudden transient episode of imbalance, lasting a few seconds. Over the next five weeks she suffered several similar episodes and two clear episodes of transient monocular visual loss (amaurosis fugax) in the left eye. On 15 February 1979 a small arterial embolus was clearly visible in the left retina on ophthalmoscopy. There were no other new cardiovascular signs. In particular there was no carotid bruit and no clinical evidence of infective endocarditis. Her haemoglobin level and white cell count were normal, and the erythrocyte sedimentation rate was $4 \mathrm{~mm}$ in the first hour. Prothrombin times measured on 9 and 23 January and 13 February had all been more than twice the control times and were comparable to levels recorded during the previous year.

Oestrogens were discontinued, and dipyridamole (Persantin) $100 \mathrm{mg}$ thrice daily started. The patient stopped taking this drug after five days, however, because of headache, which persisted despite a reduction in dose. Two months later she had experienced no further embolic symptoms and the valve prosthesis continued to function normally.

\section{Comment}

The clear temporal relationship between the ingestion of oestrogen and the occurrence of transient cerebral ischaemic attacks in this case leaves little doubt that oestrogen therapy precipitated these 